Article

\title{
Structure of the C-terminal Region of the Frizzled Receptor 1 in Detergent Micelles
}

\author{
Shovanlal Gayen ${ }^{1}$, Qingxin $\mathrm{Li}^{2}$, Young Mee Kim ${ }^{1}$ and CongBao Kang ${ }^{1, *}$ \\ 1 Experimental Therapeutics Center, the Agency for Science, Technology and Research, \\ Singapore 138669, Singapore \\ 2 Institute of Chemical \& Engineering Sciences, Agency for Science, \\ Technology and Research (A*STAR), Singapore 627833, Singapore \\ * Author to whom correspondence should be addressed; E-Mail: cbkang@etc.a-star.edu.sg; \\ Tel.: +65-6407-0602; Fax: +65-6478-8768.
}

Received: 24 June 2013; in revised form: 18 July 2013 / Accepted: 18 July 2013 /

Published: 22 July 2013

\begin{abstract}
The C-terminal domains of the Frizzleds (FZDs) contain a short conserved motif (KTXXXW). It has been demonstrated that FZDs interacted with the PDZ domain of the cytoplasmic proteins such as Dishevelled through this motif and mutations in this motif disrupted Wnt/ $\beta$-catenin signaling. We carried out structural studies for a peptide derived from the C-terminal domain of the $\mathrm{FZD}_{1}$ in different solvents using circular dichroism and solution NMR spectroscopy. Our results showed that this domain was unstructured in an aqueous solution and formed a helical structure in detergent micelles. Fluorescence studies suggested that the tryptophan residue (W630) in the motif interacted with micelles. The solution structure of the peptide in sodium dodecyl sulfate micelles was determined and an amphipathic helix was identified. This helix may have similar function to the helix 8 of other $\mathrm{G}$ protein-coupled receptors.
\end{abstract}

Keywords: helix 8; Frizzled receptor; detergent micelles; NMR

\section{Introduction}

G protein-coupled receptors (GPCRs) are a class of membrane proteins mediating important cellular signal transductions across the cell membrane. Structural studies for GPCRs have been conducted using X-ray crystallography and NMR spectroscopy [1-3]. GPCRs have a structure fold containing an 
N-terminal domain, a seven-transmembrane core domain and a C-terminal domain. Although the membrane topology of the transmembrane domains of GPCRs is similar, the N- and C-terminal regions of different GPCRs have various length and structures. GPCRs comprise a protein superfamily consisting of Class A, B, C and Class Frizzled receptors (FZDs). The FZDs contain ten members and smoothened receptor (SMO) [4]. The extracellular N-terminal domains of FZDs have a cystein-rich domain (CRD) followed by a hydrophobic linker with different lengths [5]. The CRD binds Wnt molecules that are important in embryonic development and stem cell maintenance [5].

The C-terminal regions of GPCRs interact with other proteins or help folding of the GPCRs. For class A rhodopsin-like family of GPCRs, the C-terminal region following the seventh transmembrane segment contains a helix which is amphipathic and referred as helix 8 [6]. The helix 8 normally starts after a conserved NPXXY motif in the seventh transmembrane helix. Structures of the helix 8 from different GPCRs have been studied using NMR spectroscopy [7-9]. In aqueous solutions, the peptide lacks secondary structures. In the presence of a membrane-mimicking environment such as detergent micelles, the peptide derived from the helix 8 forms a helical structure with hydrophobic residues interacting with detergent micelles [7,10]. The conformational changes in the presence and absence of membrane-mimicking environment may represent the activation of the receptor upon ligand binding [7,11]. The C-terminal domain following the transmembrane region of the FZDs have different length and sequence similarity (Figure 1). The domain has been shown to be necessary for localization of the receptors [12]. Studies have also demonstrated that there is a short conserved motif (KTXXXW) present in the C-terminal domain [13]. Mutations in this motif disrupted Wnt/ $\beta$-catenin signaling [13]. Further study has demonstrated that this motif interacted with the PDZ domain of the cytoplasmic protein-Dishevelled (Dvl) with a low binding affinity [14]. By using a peptide scanning method, two motifs of the third intracellular loop and the C-terminal region of the FZD are identified to be important for forming a stable FDZ-Dvl complex [15].

Due to the importance of the C-terminal domain of the FZDs in the Wnt/ $\beta$-catenin signaling, it will be useful to understand their structures. Compared with class A rhodopsin-like family of GPCRs, there is no NPXXY motif present in the seventh transmembrane helix of the FZDs (Figure 1). If the C-terminal domain contains a helix 8 is still unknown. In this study, we conducted structural analysis of the C-terminal region of the $\mathrm{FZD}_{1}$ consisting of 25 residues (Figure 1). Our results indicated that the peptide was not structured in an aqueous solution, but formed a helical structure in detergent micelles such as sodium dodecyl sulfate (SDS) and dodecylphosphocholine (DPC). Sequence analysis and structural studies indicated that the $\mathrm{C}$-terminal domain of $\mathrm{FZD}_{1}$ contains an amphipathic helix which is similar to other GPCRs. Residue such as Trp in this domain is interacting with micelles. Our result will be useful to understand the role of the C-terminal domains of FZDs in signal transductions.

\section{Results and Discussion}

The FZDs are unconventional GPCRs and classified as a novel family of GPCRs [4]. For the conventional GPCRs, the C-terminal domain is important for signaling and G protein coupling. Examples include rhodopsin and CB1 receptors [16,17]. The C-terminal domains of FDZs were also shown to involve in signal transduction, while its structure is still unknown. 


\subsection{Selection of the C-terminal Domain of $F Z D_{1}$}

The C-terminal domains from the FZDs have different length and sequence diversity (Figure 1). To understand the structure, we focused on the $\mathrm{FZD}_{1}$ because it has a relatively short sequence which is suitable for NMR studies. Peptide corresponding to S623 to V647 was synthesized and purified (Figure 1). Further studies were carried out to understand its structure under different conditions.

Figure 1. Sequence alignment of the C-terminal domains of the FZDs and SMO. The C-terminal domains from ten FZDs $\left(\mathrm{FZD}_{1-10}\right)$ and $\mathrm{SMO}$ were aligned using ClustalW (http://www.ebi.ac.uk/Tools/msa/clustalw2/). The conserved KTXXXW motif is highlighted in bold. The sequence used in the study is shown with a box. The protein sequences were obtained from protein knowledgebase (http://www.uniprot.org) with the following accession numbers: FZD 1 , Q9UP38; FZD 2 Q14332; FZD 3 , Q9NPG1; FZD Q9ULV1; FZD 5 , Q13467; FZD 6 , O60353; FZD 7 O75084; FZD 8 Q9H461; FZD O00144; FZD 10 , Q9ULW2; SMO, Q99835. For clarity, only a partial sequence from the $\mathrm{C}$-terminus of SMO is shown.

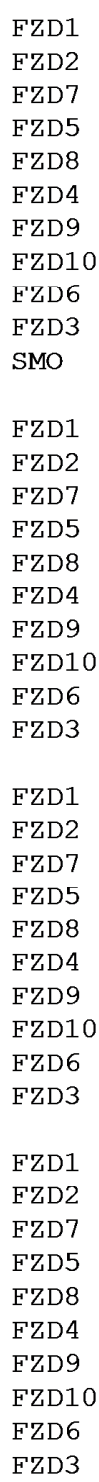

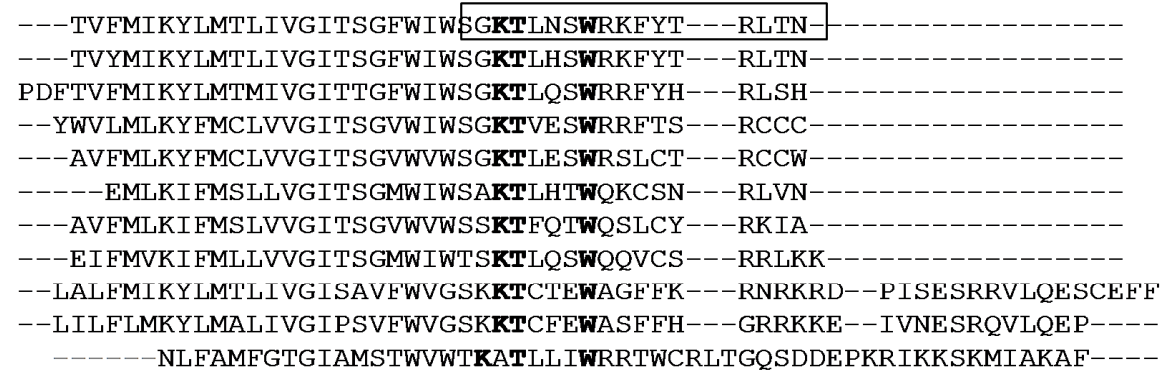

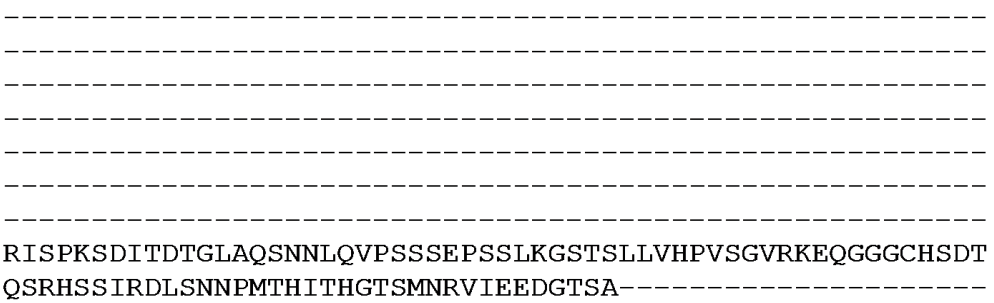




\subsection{Circular Dichroism (CD) Experiment}

Far-UV CD was first employed to examine the conformations of peptide derived from $\mathrm{FZD}_{1}$ under different conditions. CD result showed that when the peptide was prepared in an aqueous solution containing only $20 \mathrm{mM}$ sodium phosphate, $\mathrm{pH} 7.0$, the peptide was unstructured (Figure $2 \mathrm{~A}$ ). In the presence of detergent micelles such as SDS and DPC that were used as membrane mimetics [18], the CD spectra of the peptide in these two detergent micelles changed obviously with spectra characterized for an $\alpha$-helix in which double minima at 222 and $208 \mathrm{~nm}$ were observed (Figure 2A). Further data analysis using the K2D2 server demonstrated that peptide acquired similar helical conformation in these two detergent solutions. It was predicted from the K2D2 server that the helical content was approximately $70 \%$ in these two solutions. Micelle-dependent conformational change has been demonstrated for several peptides derived from GPCRs and other membrane proteins $[7,8,19]$. Our result suggested that the C-terminal region of the $\mathrm{FZD}_{1}$ could form an $\alpha$-helix in the presence of detergent micelles mimicking conditions of cell membrane proximity.

Figure 2. Effect of solvent systems on the peptide structure. (A) CD spectra of the peptide in different solutions. The CD spectra of the peptide in an aqueous solution, DPC and SDS micelles are shown with dotted, dashed and solid lines, respectively. (B) Fluorescence spectroscopy for the peptide. The emission spectra for peptide in different solutions are shown.

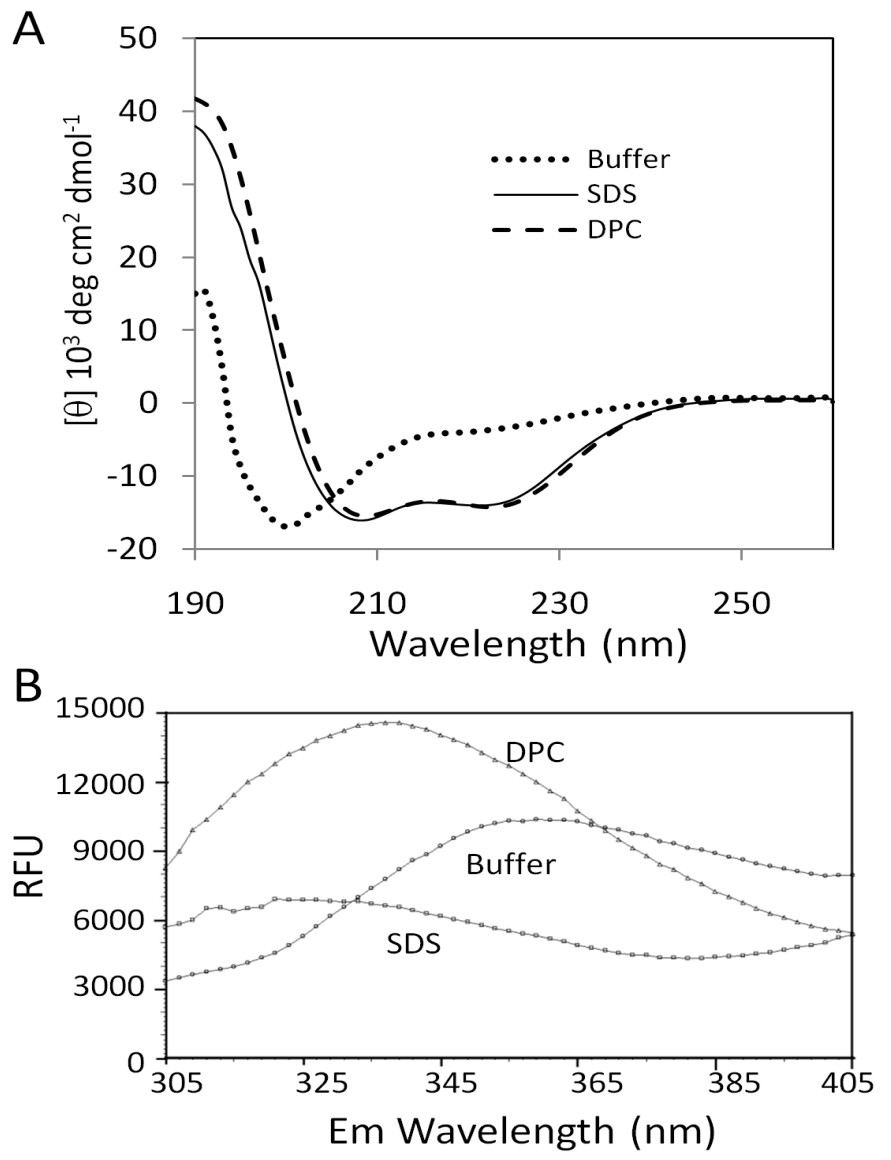




\subsection{Fluorescence Analysis}

To understand the interaction between the peptide and detergent micelles, fluorescence spectroscopy was carried out. It is known that the fluorescence emission spectrum of tryptophan residues is very sensitive to the environment, which can be used to measure residue and membrane interactions. In an aqueous solution, the emission maximum is close to $350 \mathrm{~nm}$ after excitation at $280 \mathrm{~nm}$. If the tryptophan residue is buried in a hydrophobic environment such as in the presence of micelles, the emission maximum will be shifted to a lower wavelength [20]. The peptide in the aqueous solution had an emission maximum of $350 \mathrm{~nm}$, indicating that the W630 was exposed to the solvent (Figure 2B). In the presence of the DPC micelles, the emission maximum shifted to $\sim 330 \mathrm{~nm}$, indicating that the residue W630 was buried in the DPC micelles. Although there was no clear emission maximum observed when SDS micelles were present, the disappearance of maximum at $350 \mathrm{~nm}$ indicated that the tryptophan residue was buried in or affected by SDS micelles (Figure 2B).

\subsection{NMR Study}

SDS or DPC were used as a membrane mimetic in membrane protein structural studies [18,21]. To study the structures of the peptide in the presence and absence of detergent micelles, NMR data for peptide dissolved in different solutions were collected. The peptide produced poorly dispersed spectra in DPC micelles which may due to the size of the peptide- DPC micelles (data not shown). Resonance assignments for the peptide in the aqueous solution and SDS micelles were obtained (Figure 3). Although peptide in aqueous solution exhibited dispersed cross-peaks in a ${ }^{15} \mathrm{~N}-\mathrm{HSQC}$ spectrum, further NOE connection indicated that this peptide was not structured because only $d_{\mathrm{NN}}(i, i+1)$ connections were observed (Figure 3E). This result is consistent with the CD data. When the peptide was dissolved in SDS micelles, we conducted NMR experiments at $313 \mathrm{~K}$ because the signal was improved compared to $298 \mathrm{~K}$. NOE connections indicated that residues L627 to N639 formed a helix, which was confirmed by the presence of $\alpha \mathrm{N}(i, i+3)$ and $\alpha \mathrm{N}(i, i+4)$ NOE connections (Figure 3$)$. SDS micelles have been used as a membrane-mimicking system in structural studies of several membrane proteins [18]. The data suggested that peptide may form a helix when it is close to the cell membrane.

\subsection{Structure Determination}

The structure of the peptide in SDS micelles was determined based on 104 NOEs (Table 1). Figure 4 shows the 20 superimposed structures with lowest energy. The root-mean-square deviation (RMSD) for the backbone atoms from the helix of the twenty structures was $0.4 \AA$ (Table 1). The restraints used in this study and the structural analysis using PROCHECK-NMR [22] are shown in Table 1. Residues L627 to N639 formed a helix and the C-terminus of this peptide was not structured (Figure 4). Helix wheel representation for this helix indicated that it behaves like an amphipathic helix (Figure 4). The hydrophobic residues such as $\mathrm{W}, \mathrm{L}$ and $\mathrm{Y}$ are facing one side and charged residues such as $\mathrm{R}$ and $\mathrm{N}$ are facing the other side (Figure 4). These hydrophobic residues may be important for interacting micelles, for example, the Trp residue was buried in the micelles from our fluorescence experiment (Figure 2B).

Solvent-induced conformational changes have been observed in C-terminal domains of other GPCRs such as human $\beta 2$ adrenergic receptor [8] and CB1 cannabinoid receptor [7]. In those 
receptors, their C-terminal domains contain a helix 8 following the seventh transmembrane helix. Helix 8 in GPCRs may serve as a membrane anchor because the helix is amphipathic and contains palmitoylation sites that can stabilize the interaction with membrane. The conformation of the helix may be regulated by allosteric modulators binding to the seven-transmembrane core domain. After activation by an allosteric ligand, the helix 8 may become exposed to form a disordered structure to expose its binding site for other proteins [7]. In GPCRs such as rhodopsin, the hydrophobic residues in this motif may stack against each other to keep the receptor in a pre-receptive state [6,23]. Unlike other GPCRs, there is no NPXXY motif present in the seventh transmembrane helix of FZDsFrom our studies, it was demonstrated that the C-terminal domain of $\mathrm{FZD}_{1}$ had similar characteristics to the helix 8 from other GPCRs such as CB1 receptor.

Figure 3. NMR analysis of the C-terminal region of the $\mathrm{FZD}_{1}$. Assignments for the ${ }^{15} \mathrm{~N}-\mathrm{HSQC}$ spectra of the peptide in the phosphate buffer (A) and in SDS micelles (B) are shown. NOESY spectra of the peptide in the phosphate buffer $(\mathbf{C})$ and in SDS micelles (E) are shown. The NOEs between different residues are labeled residues name and sequence number. The NOE connections of the peptide in the aqueous solution (E) and in SDS (F) micelles are shown.
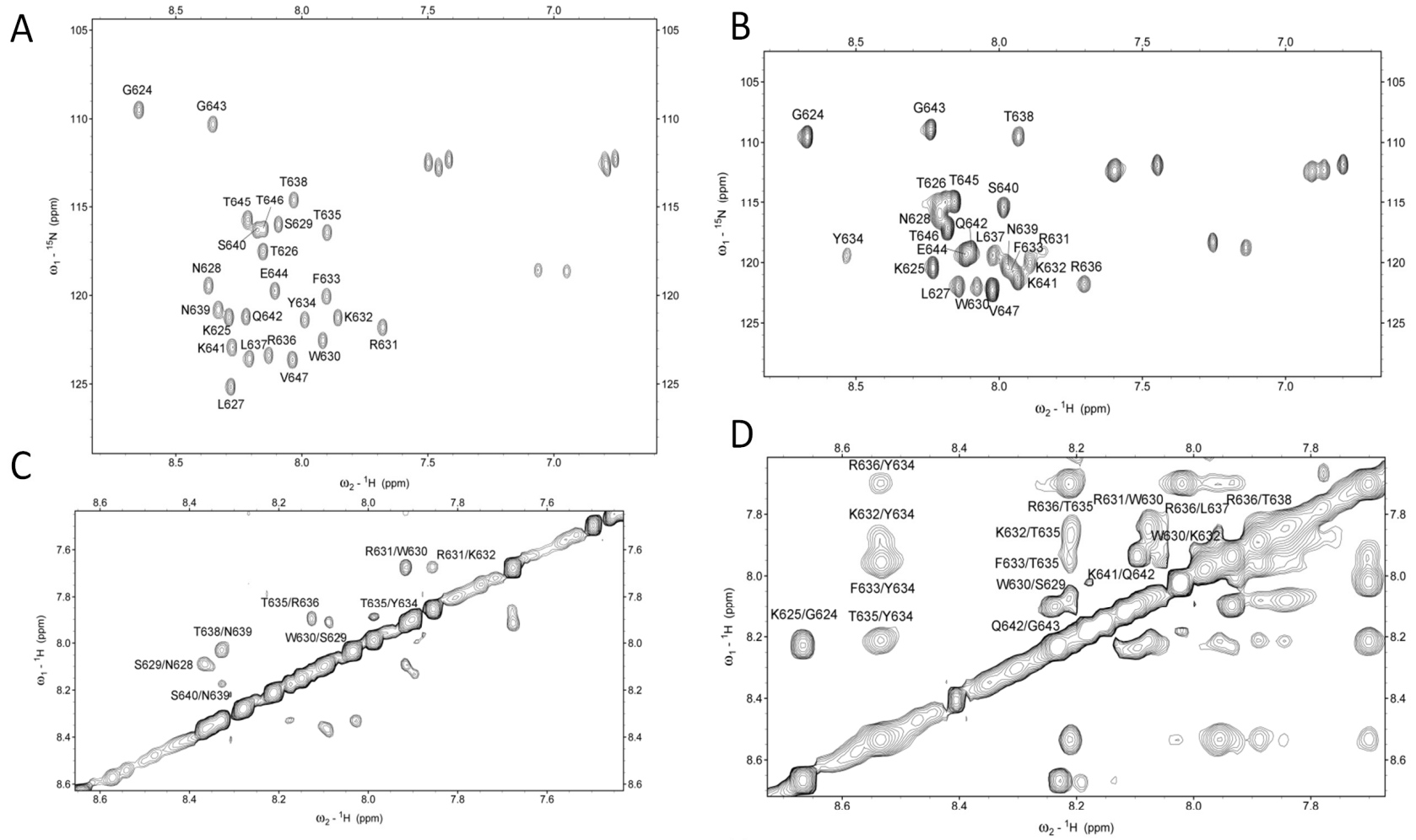

$E$
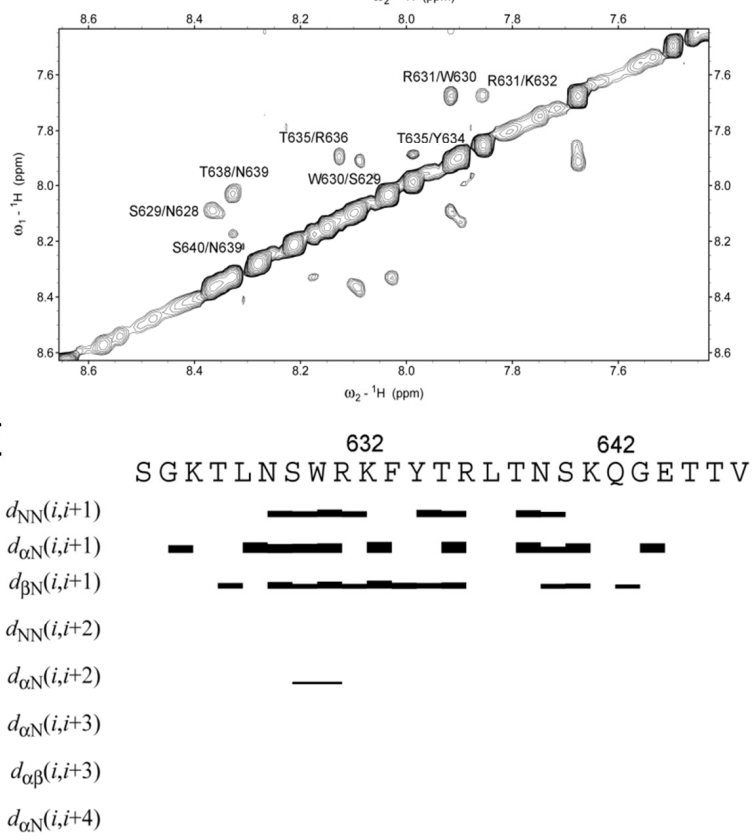

$\mathrm{F}$
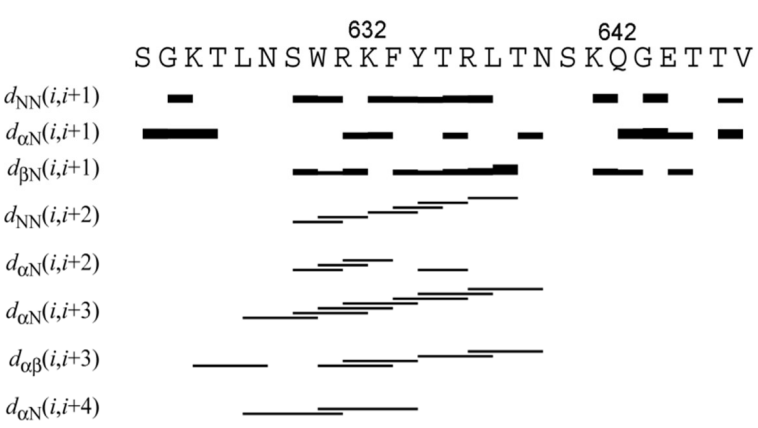
Table 1. Experimental distance and statistics for the 20 structures determined in SDS micelles.

\begin{tabular}{lc}
\hline Restraints & \\
\hline Intraresidue NOEs & 71 \\
Sequential NOEs (i to i+1) & 65 \\
Medium range NOEs (i to i $+2,3,4)$ & 39 \\
Dihedral angle restraints & 0 \\
Number of NOE violations $>0.5 \AA$ & 0 \\
Ramachandran plot (\%) & \\
Residues in most favored regions & 55.8 \\
Residues in additional allowed regions & 38.8 \\
Residues in generously allowed regions & 5.7 \\
Residues in disallowed regions & 0 \\
Structural statistics & \\
RMSD for backbone atoms (6-17) & $0.4 \AA$ \\
RMSD for heavy atoms (6-17) & $0.86 \AA$ \\
\hline
\end{tabular}

Figure 4. Structure of the peptide in SDS micelles. (A) Superimposed structures with lowest energy. Only backbone atom traces are shown. (B) Cartoon representation of the structure. The Trp and Tyr residues are shown in sticks. (C) Surface representation of the peptide structure. The charge representation of the helical surface and the figures were made using PyMOL (http://www.pymol.org). (D) Helix wheel representation for the helix.
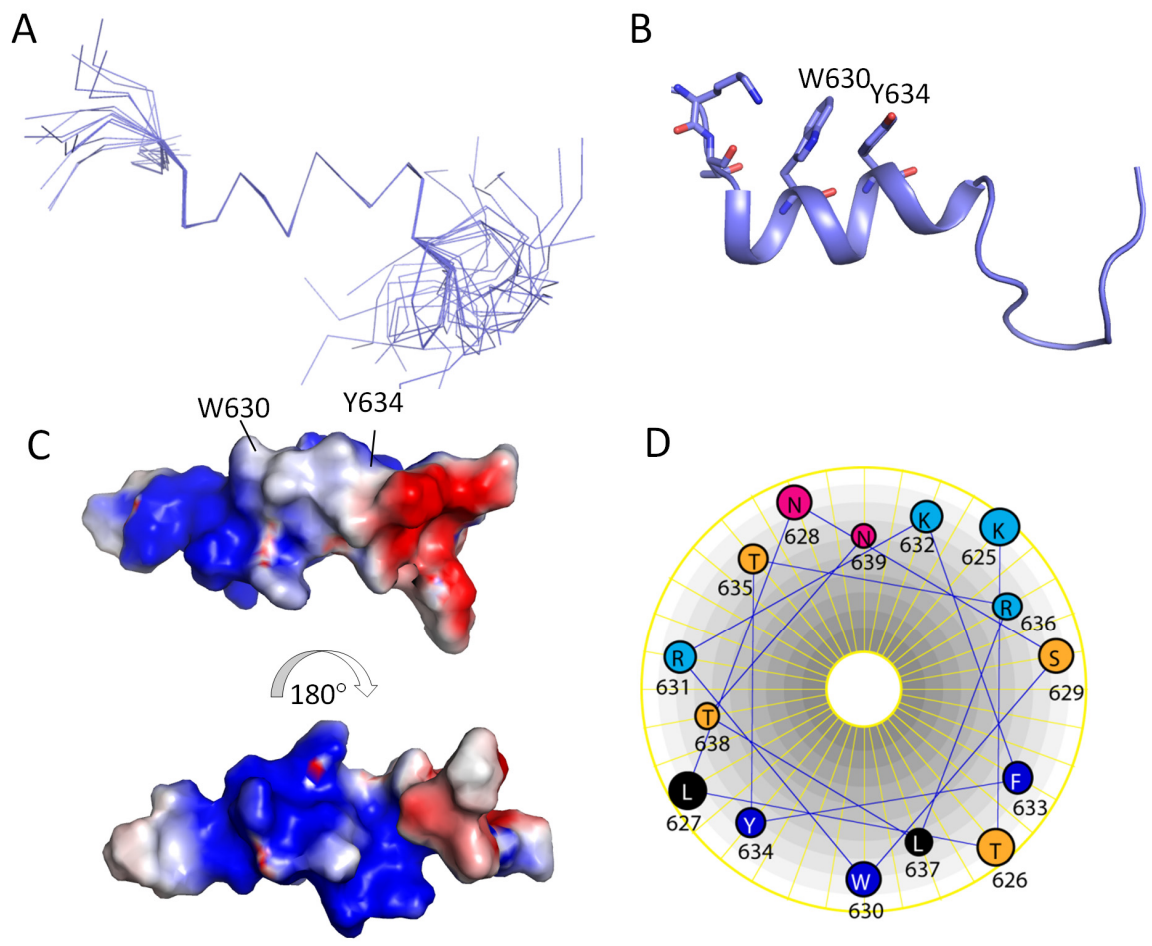

The structure of the smoothened (SMO) receptor which shows sequence similarity to the FZDs was determined using X-ray crystallography. SMO also has a conserved KATXXXW motif at the C-terminus [24] and this motif has been described to stabilize the receptor by packing parallel to the membrane (Figure 5). The tryptophan residue (W545) localized at the membrane interface, which is similar to what we observed in the C-terminal domain of the FZD ${ }_{1}$. The C-terminal domain of FZDs 
contains a conserved KTXXXW motif lacking an alanine residue for PDZ domain interactions [13] (Figure 1). From our study, this motif had a tendency to form a helix. Although the KT residues within this motif are not helical under current conditions, this may arise from the fact that this peptide did not include the seventh transmembrane helix. The tryptophan residue (W630) is involved in the micelle interactions, which was confirmed from the fluorescence study (Figure 2B). Mutations in this motif may disrupt the helical structure or affect membrane interaction [13], which in turn will affect the signal transduction or binding to Dvl [15]. All these results suggest that the C-terminal domains of FZDs might have similar role to those of the conventional GPCRs. There is a cystein residue present in the helix 8 of GPCRs. This cystein can be modified by palmitoylation, which could serve as a lipid anchor. As there is no cystein present in the C-terminus of FZDs, this tryptophan residue may act as a lipid anchor to stabilize the interaction with membrane. Under different conditions, this domain interacts with Dvl or membrane, which will produce different signals for the downstream pathways. Previous studies have proposed that the helix 8 in GPCRs is involved in G-protein coupling and GPCR activation [10]. The sequence similarity within FZDs suggested that the helix 8 may be a common structural feature within this family (Figure 1). This region might have the similar functional roles to the helix 8 of the classical GPCRs. Our study provided structural basis to understand its function.

Figure 5. Role of the Trp residues in membrane binding. A. Crystal structure of the SMO receptor. B. Homology model of the $\mathrm{FZD}_{1}$ using the crystal structure of the SMO receptor as a template [25]. The possible membrane interface is shown in dash line. The residues in both structures are shown in sticks.

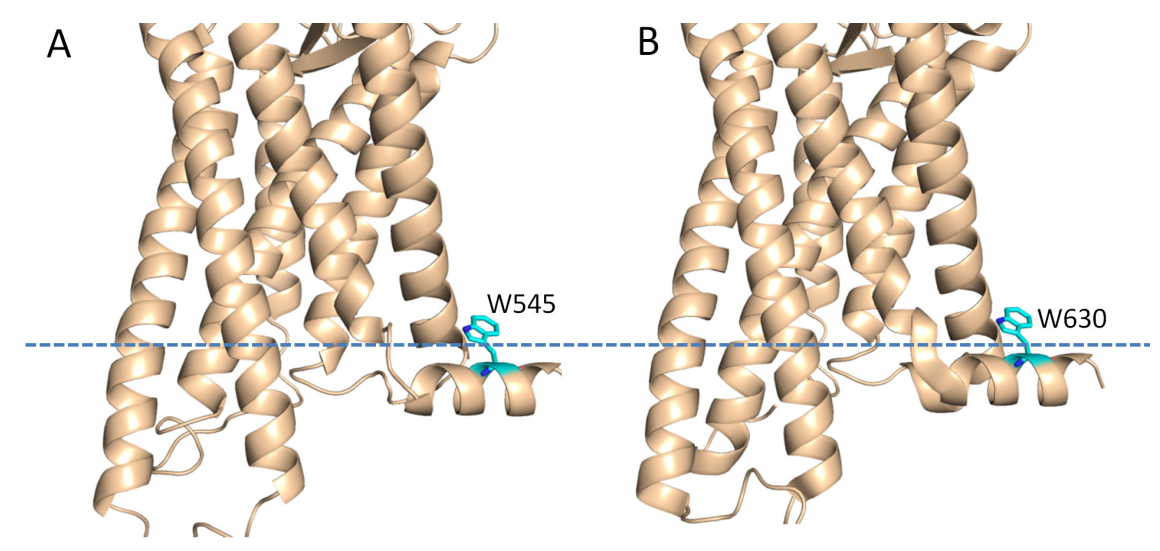

\section{Experimental}

\subsection{Sample Preparation}

The peptide sequence was SGKTLNSWRKFYTRLTNSKQGETTV and was derived from the C-terminal domain of the $\mathrm{FZD}_{1}$ corresponding to residue 623 to 647 . This peptide was synthesized and purified from GL Biochem Ltd (Shanghai, China) with more than 95\% purity. The peptide was prepared to a concentration of $5 \mathrm{mg} / \mathrm{mL}$ in a buffer containing $20 \mathrm{mM}$ sodium phosphate, $\mathrm{pH} 7.0$ or same buffer that contained $2 \%$ deuterated DPC or SDS. The samples with $10 \% \mathrm{D}_{2} \mathrm{O}$ were transferred into 3-mm NMR tubes for data acquisition. 


\subsection{Circular Dichroism (CD) Spectroscopy}

For secondary structural analysis, samples were diluted to $0.2 \mathrm{mg}$ of peptide per $\mathrm{ml}$ for $\mathrm{CD}$ analysis as described previously [19]. Peptide was dissolved in sodium phosphate buffer in the present and absence of detergent micelles. All the CD experiments were conducted using a Chirascan ${ }^{\mathrm{TM}}$ Circular Dichroism Spectrometer at $25{ }^{\circ} \mathrm{C}$. The CD spectra were recorded in a continuous mode with a 1-nm data pitch. The secondary structure was analyzed using the K2D2 server (http://www.ogic.ca/projects/k2d2/).

\subsection{Fluorescence Spectroscopy}

The effect of the solvent on the trytophan residue was measured using the similar method described [20]. The fluorescence emission spectra were measured in a 96-well plate. Peptide was dissolved in the buffer to a concentration of $0.1 \mathrm{mg} / \mathrm{mL}$. Samples with $100 \mu \mathrm{L}$ volume in the presence and absence of micelles were subjected to analysis. Excitation wavelength was $280 \mathrm{~nm}$ and the emission was scanned from 305 to $405 \mathrm{~nm}$. Experiment was carried out at $37^{\circ} \mathrm{C}$.

\subsection{NMR Experiments}

The NMR data were collected on a Bruker Avance II spectrometer with a proton frequency of $700 \mathrm{MHz}$ equipped with a cryoprobe. A total correlation spectroscopy (TOCSY) experiment was recorded with a mixing time of $80 \mathrm{~ms}$ [26]. Two-dimensional (2D) nuclear overhauser effect spectroscopy (NOESY) experiments were recorded with mixing times of $100 \mathrm{~ms}, 200 \mathrm{~ms}$ and $300 \mathrm{~ms}$, respectively. The experiments for peptide in sodium phosphate buffer were performed at $298 \mathrm{~K}$. In the presence of micelles, the TOCSY and NOESY spectra were recorded at $313 \mathrm{~K}$ to increase the resolution. Proton chemical shift values were referenced directly to 4, 4-dimethyl-4-silapentane-1-sulfonic acid (DSS). All of the spectra were processed using Topspin 2.1 and analyzed using Sparky (http://www.cgl.ucsf.edu/ home/sparky/).

\subsection{Resonance Assignment and Structure Determination}

The resonance assignment for the peptide was obtained using the procedure including the identification of spin systems in a TOCSY spectrum and spin connections in a NOESY spectrum [26,27]. The sequential connectivity of the residues was confirmed based on the connectivity in the HN-HN or Ha-HN region. A ${ }^{1} \mathrm{H}^{15} \mathrm{~N}$ heteronuclear single quantum correlation spectroscopy (HSQC) in ${ }^{15} \mathrm{~N}$ natural abundance was acquired and processed to facilitate resonance assignment. The NOE peaks picked from a NOESY spectrum with a mixing time of $200 \mathrm{~ms}$ were integrated for structural determination. The peaks were assigned manually and calibrated to distances using CYANA 2.1 for structural determination [28]. Structure determination for peptide in SDS micelles was conducted as described previously [26]. The structure was calculated using torsion angle dynamics simulated annealing as implemented in CYANA using NOE restraints [26,28]. One hundred structures were calculated and twenty of the structures with lowest energies were analyzed with MOLMOL [29] and displayed using PyMOL (http://www.pymol.org). 


\section{Conclusions}

In summary, we carried out structural studies for a peptide derived from the C-terminal domain FZD $_{1}$. Our results show that the peptide adopted different conformations under different solvent conditions. In the aqueous solution, it was not structured. It formed a helical structure when a membranemimicking detergent was present. The tryptophan residue (W630) in the KTXXXW motif may be important for membrane interaction.

\section{Acknowledgments}

CK appreciates the support from A*STAR Investigatorship and A*STAR JCO grant (10/03/FG/06/06).

\section{Conflict of Interest}

The authors declare no conflict of interest.

\section{References}

1. Katritch, V.; Cherezov, V.; Stevens, R.C. Diversity and modularity of G protein-coupled receptor structures. Trends Pharmacol. Sci. 2012, 33, 17-27.

2. Park, S.H.; Das, B.B.; Casagrande, F.; Tian, Y.; Nothnagel, H.J.; Chu, M.; Kiefer, H.; Maier, K.; De Angelis, A.A.; Marassi, F.M.; et al. Structure of the chemokine receptor CXCR1 in phospholipid bilayers. Nature 2012, 491, 779-783.

3. Venkatakrishnan, A.J.; Deupi, X.; Lebon, G.; Tate, C.G.; Schertler, G.F.; Babu, M.M. Molecular signatures of G-protein-coupled receptors. Nature 2013, 494, 185-194.

4. Schulte, G.; Bryja, V. The Frizzled family of unconventional G-protein-coupled receptors. Trends Pharmacol. Sci. 2007, 28, 518-525.

5. Huang, H.C.; Klein, P.S. The Frizzled family: Receptors for multiple signal transduction pathways. Genome Biol. 2004, 5, 234.

6. Huynh, J.; Thomas, W.G.; Aguilar, M.I.; Pattenden, L.K. Role of helix 8 in G protein-coupled receptors based on structure-function studies on the type 1 angiotensin receptor. Mol. Cell Endocrinol. 2009, 302, 118-127.

7. Choi, G.; Guo, J.; Makriyannis, A. The conformation of the cytoplasmic helix 8 of the CB1 cannabinoid receptor using NMR and circular dichroism. Biochim. Biophys. Acta 2005, 1668, 1-9.

8. Katragadda, M.; Maciejewski, M.W.; Yeagle, P.L. Structural studies of the putative helix 8 in the human beta(2) adrenergic receptor: An NMR study. Biochim. Biophys. Acta 2004, 1663, 74-81.

9. Tyukhtenko, S.; Tiburu, E.K.; Deshmukh, L.; Vinogradova, O.; Janero, D.R.; Makriyannis, A. NMR solution structure of human cannabinoid receptor-1 helix 7/8 peptide: Candidate electrostatic interactions and microdomain formation. Biochem. Biophys. Res. Commun. 2009, 390, 441-446.

10. Bruno, A.; Costantino, G.; de Fabritiis, G.; Pastor, M.; Selent, J. Membrane-sensitive conformational states of helix 8 in the metabotropic Glu2 receptor, a class C GPCR. PLoS One 2012, 7, e42023. 
11. Wess, J.; Han, S.J.; Kim, S.K.; Jacobson, K.A.; Li, J.H. Conformational changes involved in G-protein-coupled-receptor activation. Trends Pharmacol. Sci. 2008, 29, 616-625.

12. Wu, J.; Klein, T.J.; Mlodzik, M. Subcellular localization of frizzled receptors, Mediated by their cytoplasmic tails, Regulates signaling pathway specificity. PLoS Biol. 2004, 2, E158.

13. Umbhauer, M.; Djiane, A.; Goisset, C.; Penzo-Mendez, A.; Riou, J.F.; Boucaut, J.C.; Shi, D.L. The C-terminal cytoplasmic Lys-thr-X-X-X-Trp motif in frizzled receptors mediates Wnt/betacatenin signalling. EMBO J. 2000, 19, 4944-4954.

14. Wong, H.C.; Bourdelas, A.; Krauss, A.; Lee, H.J.; Shao, Y.; Wu, D.; Mlodzik, M.; Shi, D.L.; Zheng, J. Direct binding of the PDZ domain of Dishevelled to a conserved internal sequence in the C-terminal region of Frizzled. Mol. Cell 2003, 12, 1251-1260.

15. Tauriello, D.V.; Jordens, I.; Kirchner, K.; Slootstra, J.W.; Kruitwagen, T.; Bouwman, B.A.; Noutsou, M.; Rudiger, S.G.; Schwamborn, K.; Schambony, A.; et al. Wnt/beta-catenin signaling requires interaction of the Dishevelled DEP domain and $\mathrm{C}$ terminus with a discontinuous motif in Frizzled. Proc. Natl. Acad. Sci. USA 2012, 109, E812-E820.

16. Ernst, O.P.; Meyer, C.K.; Marin, E.P.; Henklein, P.; Fu, W.Y.; Sakmar, T.P.; Hofmann, K.P. Mutation of the fourth cytoplasmic loop of rhodopsin affects binding of transducin and peptides derived from the carboxyl-terminal sequences of transducin alpha and gamma subunits. J. Biol. Chem. 2000, 275, 1937-1943.

17. Mukhopadhyay, S.; Cowsik, S.M.; Lynn, A.M.; Welsh, W.J.; Howlett, A.C. Regulation of Gi by the $\mathrm{CB} 1$ cannabinoid receptor C-terminal juxtamembrane region: Structural requirements determined by peptide analysis. Biochemistry 1999, 38, 3447-3455.

18. Kang, C.; Li, Q. Solution NMR study of integral membrane proteins. Curr. Opin. Chem. Biol. 2011, 15, 560-569.

19. Gayen, S.; Li, Q.; Kang, C. The solution structure of the S4-S5 linker of the hERG potassium channel. J. Pept. Sci. 2011, 18, 140-145.

20. Conner, M.; Hicks, M.R.; Dafforn, T.; Knowles, T.J.; Ludwig, C.; Staddon, S.; Overduin, M.; Gunther, U.L.; Thome, J.; Wheatley, M.; et al. Functional and biophysical analysis of the Cterminus of the CGRP-receptor; a family B GPCR. Biochemistry 2008, 47, 8434-8444.

21. Gayen, S.; Li, Q.; Kang, C.B. Solution NMR study of the transmembrane domain of single-span membrane proteins: Opportunities and strategies. Curr. Protein Pept. Sci. 2012, 13, 585-600.

22. Laskowski, R.A.; Rullmannn, J.A.; MacArthur, M.W.; Kaptein, R.; Thornton, J.M. AQUA and PROCHECK-NMR: programs for checking the quality of protein structures solved by NMR. J. Biomol. NMR 1996, 8, 477-486.

23. Fritze, O.; Filipek, S.; Kuksa, V.; Palczewski, K.; Hofmann, K.P.; Ernst, O.P. Role of the conserved $\operatorname{NPxxY}(\mathrm{x}) 5,6 \mathrm{~F}$ motif in the rhodopsin ground state and during activation. Proc. Natl. Acad. Sci. USA 2003, 100, 2290-2295.

24. Wang, C.; Wu, H.; Katritch, V.; Han, G.W.; Huang, X.P.; Liu, W.; Siu, F.Y.; Roth, B.L.; Cherezov, V.; Stevens, R.C. Structure of the human smoothened receptor bound to an antitumour agent. Nature 2013, 497, 338-343.

25. Arnold, K.; Bordoli, L.; Kopp, J.; Schwede, T. The SWISS-MODEL workspace: A web-based environment for protein structure homology modelling. Bioinformatics 2006, 22, 195-201. 
26. Gayen, S.; Kang, C. Solution structure of a human minimembrane protein Ost 4 , a subunit of the oligosaccharyltransferase complex. Biochem. Biophys. Res. Commun. 2011, 409, 572-576.

27. Wüthrich, K. NMR of Proteins and Nucleic Acids; Wiley: New York, NY, USA; 1986.

28. Guntert, P. Automated NMR structure calculation with CYANA. Methods Mol. Biol. 2004, 278, 353-378.

29. Koradi, R.; Billeter, M.; Wuthrich, K. MOLMOL: A program for display and analysis of macromolecular structures. J. Mol. Graph. 1996, 14, 29-32, 51-55.

Sample Availability: Commercial available.

(C) 2013 by the authors; licensee MDPI, Basel, Switzerland. This article is an open access article distributed under the terms and conditions of the Creative Commons Attribution license (http://creativecommons.org/licenses/by/3.0/). 